OPEN

SUBJECT AREAS:

BIOMATERIALS -

PROTEINS

PROTEOMICS

PROTEOMIC ANALYSIS

Received

17 March 2014

Accepted

9 June 2014

Published

30 June 2014

Correspondence and requests for materials should be addressed to

L.H.Z. (lihuazhang@ dicp.ac.cn)

\section{Preparation of protein imprinted} materials by hierarchical imprinting techniques and application in selective depletion of albumin from human serum

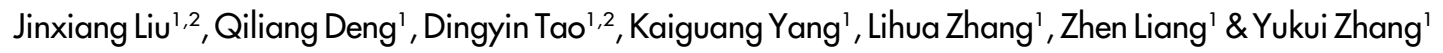

\begin{abstract}
${ }^{1}$ National Chromatographic R. \& A. Center, Key Laboratory of Separation Science for Analytical Chemistry, Dalian Institute of Chemical Physics, Chinese Academy of Sciences, Dalian 1 16023, China, ${ }^{2}$ University of Chinese Academy of Sciences, Beijing 100049, China.
\end{abstract}

Hierarchical imprinting was developed to prepare the protein imprinted materials, as the artificial antibody, for the selective depletion of HSA from the human serum proteome. Porcine serum albumin (PSA) was employed as the dummy template for the fabrication of the recognition sites. To demonstrate the advantages of the hierarchical imprinting, molecularly imprinted polymers prepared by hierarchical imprinting technique (h-MIPs) were compared with those obtained by bulk imprinting (b-MIPs), in terms of the binding capacity, adsorption kinetics, selectivity and synthesis reproducibility. The binding capacity of h-MIPs could reach $12 \mathrm{mg} \mathrm{g}^{-1}$. And saturation binding could be reached in less than $20 \mathrm{~min}$ for the h-MIPs. In the protein mixture, h-MIPs exhibit excellent selectivity for PSA, with imprinting factors as about 3.6, much higher than those for non-template proteins. For the proteomic application, the identified protein group number in serum treated by h-MIPs was increased to 422 , which is $21 \%$ higher than that obtained from the original serum, meanwhile the identified protein group number for the Albumin Removal kit was only 376. The results demonstrate that protein imprinted polymers prepared by hierarchical imprinting technique, might become the artificial antibodies for the selective depletion of high abundance proteins in proteome study.

ecently, molecular imprinted polymers (MIPs), a kind of artificial synthetic materials with specific recognition capacity for target molecules, have attracted much attention ${ }^{1-3}$. Although MIPs for small molecules recognition have been successfully applied in solid-phase extraction (SPE), chiral separation, chemical sensor, enzyme-like catalysis, drug delivery and library screening, the preparation of biomacromolcule, such as proteins, imprinted polymers are rather difficult $t^{4-8}$. The traditional molecular imprinting polymers were prepared by bulk imprinting. However, some obstacles, such as poor mass transfer and recognition sites destruction due to grinding after polymerization, still existed, and hindered the application of $\mathrm{MIPs}^{7,9,10}$. In order to overcome these disadvantages of traditional monolithic imprinting materials, recently, surface imprinting technique has been employed for protein imprinting, by which recognition sites were formed on the material surface ${ }^{11}$.

Hierarchical imprinting is a new kind of surface imprinting technique that has been successfully applied in small molecules imprinting ${ }^{12}$. Through polymerization in porous silica, regular sized MIPs particles were prepared, and showed superiority of good selectivity, fast mass transfer and improved binding capacity ${ }^{12}$. However, the preparation of protein imprinted polymers by such a technique was limited in the proteomics study due to the lack of pure proteins, especially low-abundance proteins in proteomics, as templates ${ }^{13,14}$.

With the recent development of proteome techniques, the interest in finding biomarkers from human plasma for clinical diagnosis has gained new momentum ${ }^{15-17}$. However, the wide dynamic range of proteins in abundance, over $10^{10}$, brings great challenges to discover low abundance proteins with significant biological functions ${ }^{18}$. Among known high abundance proteins, HSA occupies approximately $60 \%$ of the total protein mass in serum. Therefore, the selective depletion of HSA has been paid much attention. Among various developed methods, antibody technique has been proven the most effective on ${ }^{18}$. With the consideration of wonderful selectivity of MIPs, we explored the possibility of removing high abundance protein, HSA, by MIPs. 
(a)

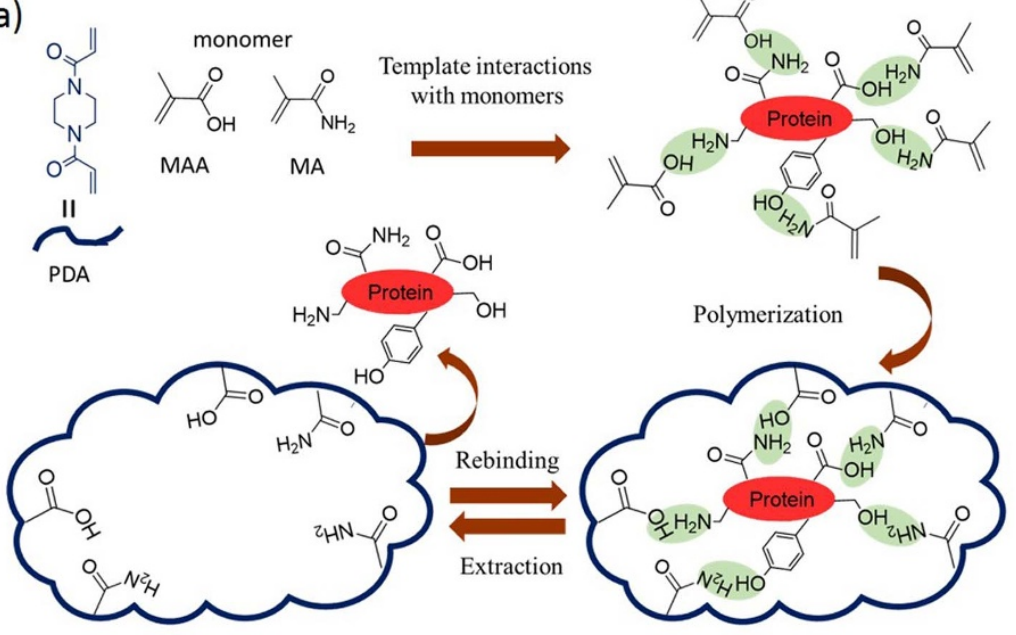

(b)

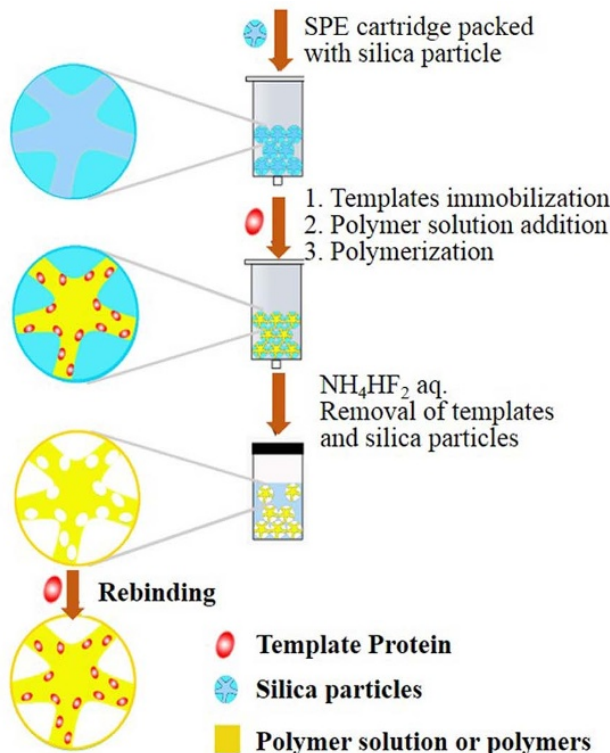

Figure $1 \mid$ Schematic illustrating the interaction between monomers and template protein (a) and the synthesis of protein imprinted polymers by hierarchical imprinting (b).

Herein, with porcine serum albumin (PSA), an analog to human serum albumin (HSA), with $80 \%$ sequence homology, as well as similar three-dimensional structure, active sites and binding domains, as the template, protein imprinted materials were prepared by hierarchical imprinting technique, and successfully applied for the selective depletion of HSA from human serum. To demonstrate the advantages of hierarchical imprinting, MIPs prepared by hierarchical imprinting technique were compared with those obtained by in bulk imprinting, in terms of the binding capacity, adsorption kinetics, selectivity and synthesis reproducibility.

\section{Experimental Section}

Reagents and instrumentation. Porcine serum albumin (PSA), ribonuclease B (RNB), cytochrome C (CYC), $\beta$-lactoglobulin (LACT) and TPCK-treated trypsin (bovine pancreas) were purchased from Sigma (St. Louis, MO, USA). Methacrylamide (MA), methacrylic acid (MAA), piperazine diarylamide (PDA), $\mathrm{N}, \mathrm{N}, \mathrm{N}^{\prime}, \mathrm{N}^{\prime}$-tetramethylethylenediamine (TEMED) and ammonium persulfate (APS) were obtained from Acros Organics (Fair Lawn, NJ, USA). Sodium dodecyl sulfate (SDS) was obtained from Promega (Madison, Wisconsin, USA). HPLC-grade acetonitrile was purchased from Merck (Darmstadt, Germany). Silica microshperes $(5 \mu \mathrm{m}, 1000 \AA)$ were bought from Fuji Sodium Silicate (Kasugai, Japan). Water was purified by a Milli-Q system (Millipore, Molsheim, France). 1,4Dithio-DL-threitol (DTT) and iodoacetamide (IAA) were purchased from Amresco (Solon, OH, USA). All inorganic reagents were analytical-reagent grade, and all the other solvents were HPLC grade. Chromatographic measurements were performed using an LC-20AD Series HPLC instrument equipped with a binary high-pressure pump, a vacuum degasser, and a UV-VIS detector and wavelength was set at $214 \mathrm{~nm}$ (Shimadzu, Kyoto, Japan). Surveyor MS pump and LTQ linear ion trap mass spectrometer were obtained from Thermo-Fisher (San Jose, CA, USA).

Preparation of bulk imprinted polymers. For both the bulk imprinted polymers and hierarchical imprinted microspheres, MA and MAA, were chosen as the functional monomers, while PDA, with favorable hydrophilicity and biological compatibility, was chosen as the crosslinker ${ }^{14}$. The MA and MAA provide carboxyl group, amide group and hydroxyl group, which could interact with the functional groups, such as amino group, carboxyl group and hydroxyl group, on the template proteins through the hydrogen interactions to form the recognition sites ${ }^{7}$, as shown in Fig. 1a.

For bulk polymerization, PSA (19.2 mg), MA (270.6 mg), MAA (9.5 mg), PDA (432.0 mg), ammonium sulfate $(234.0 \mathrm{mg}$ ) and Tween-20 (20 mg) were dissolved in the mixture of $4 \mathrm{~mL}$ of phosphate buffer $(10 \mathrm{mM}, \mathrm{pH} 6.5)$ deoxidized with nitrogen and $1 \mathrm{~mL}$ of acetonitrile. After the addition of $15 \mu \mathrm{L}$ of TEMED and $20 \mu \mathrm{L}$ of APS $(10 \% \mathrm{w} / \mathrm{v})$, the polymerization proceeded overnight. The formed polymer was washed with $10 \%(\mathrm{v} / \mathrm{v})$ acetic acid containing $10 \%$ (w/v) SDS to remove PSA, and then rinsed thoroughly with phosphate buffer until the $\mathrm{pH}$ of the effluent reached the value of 5.0, and SDS was not detected upon the addition of potassium chloride. After ground, the particles were ready for the further study. The prepared MIPs were named as b-MIPs. In addition, non-imprinted polymers (NIPs), named as b-NIPs, were prepared by the same protocol, but without the addition of PSA.

Preparation of hierarchical imprinted microspheres. For hierarchical imprinting, as shown in Fig. $1 \mathrm{~b}, 0.5 \mathrm{~g}$ of silica microspheres were dispersed in phosphate buffer $(10 \mathrm{mM}$; pH 6.5), and packed into an SPE cartridge with care to avoid bubble formation. After the residual buffer was removed under vacuum, $35 \mathrm{mg}$ PSA was dissolved in $5 \mathrm{~mL}$ phosphate buffer $(10 \mathrm{mM}$; $\mathrm{pH} 6.5)$, added into the cartridge, and vacuumed by a pump until PSA was detected in the filtrate. Subsequently, phosphate buffer was added to remove the unbound PSA. Then, MA (270 mg), MAA (9.5 mg), PDA (432 $\mathrm{mg})$, Tween-20 (20 mg) and ammonium sulfate $(234 \mathrm{mg})$ were dissolved in $4 \mathrm{~mL}$ of phosphate buffer deoxidized with nitrogen and $1 \mathrm{~mL}$ of acetonitrile, followed by the addition of $20 \mu \mathrm{L} 10 \%(\mathrm{w} /$ v) APS and $14 \mu \mathrm{L}$ TEMED. Rapidly, $2 \mathrm{~mL}$ of the solution was pushed through silica beds by a vacuum pump, and polymerized within the pores of silica overnight at room temperature. Finally, silica beads were etched with $3 \mathrm{M} \mathrm{NH} \mathrm{NHF}_{2}$ overnight, and PSA was further removed by flushed with $10 \mathrm{~mL}$ of $10 \%(\mathrm{v} / \mathrm{v})$ acetic acid containing $10 \%(w / v)$ SDS. The prepared MIPs were named as h-MIPs. For comparison, non-imprinted polymers, named as h-NIPs, were prepared with the same protocol, but without PSA added. The morphology, gravimetric yield and swelling ratio of the imprinted particles were measured, as shown in the Supporting Information. 

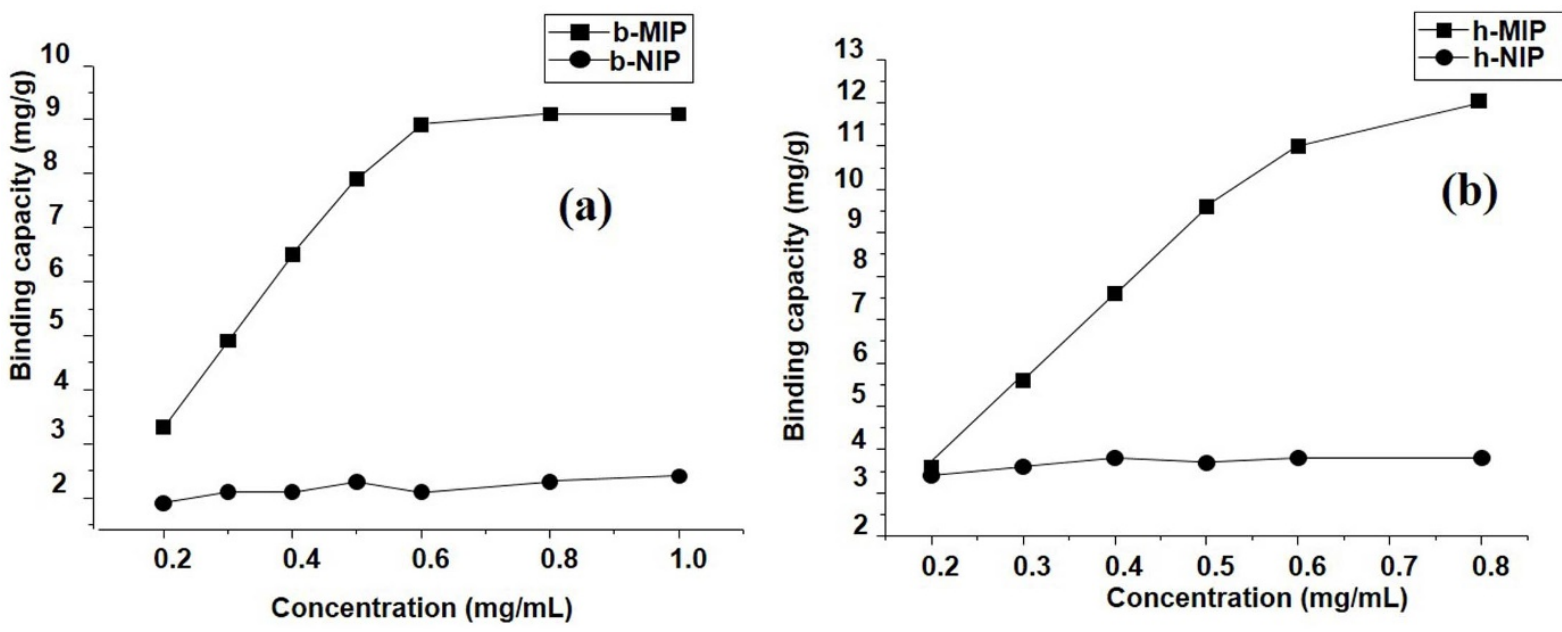

Figure $2 \mid$ Binding capacity of b-MIPs and b-NIPs (a), as well as h-MIPs and h-NIPs (b).

Binding capacity experiment. Fifty milligrams of MIPs or NIPs were incubated with $1 \mathrm{~mL}$ of PSA solution $(10 \mathrm{mM}$ phosphate buffer; $\mathrm{pH}$ 5.0) at different concentrations for $24 \mathrm{~h}$. The supernatant was collected, and the residual protein concentration was measured by HPLC.

Competitive binding experiment. Fifty milligrams of MIPs or NIPs were incubated with $1 \mathrm{~mL}$ protein mixture $(10 \mathrm{mM}$ phosphate buffer; $\mathrm{pH} 5.0$ ) for $24 \mathrm{~h}$, which was composed of PSA, RNB, CYC and LACT, with each protein concentration as $0.4 \mathrm{mg} / \mathrm{mL}$. The supernatant was collected, and the residual protein concentration was measured by HPLC.

Kinetic experiments. Fifty milligrams of MIPs were incubated with $1 \mathrm{~mL}$ of $0.5 \mathrm{mg} / \mathrm{mL}$ PSA solution ( $10 \mathrm{mM}$ phosphate buffer; $\mathrm{pH} 5.0$ ) for $24 \mathrm{~h}$. With the time intervals ranging from $5 \mathrm{~min}$ to $24 \mathrm{~h}$, the supernatant was collected, and the residual protein concentration was measured by HPLC.

Reproducibility experiment. Fifty milligrams of MIPs were incubated with $1 \mathrm{~mL}$ 4-protein-mixture solution $(10 \mathrm{mM}$ phosphate buffer; $\mathrm{pH} \mathrm{5.0)}$ for $24 \mathrm{~h}$. The supernatant was collected, and the residual protein concentration was measured by HPLC.

Depletion of albumin from human serum. Fifty milligram of hMIPs were incubated with $1 \mathrm{~mL}$ of $0.5 \mathrm{mg} / \mathrm{mL}$ serum solution (diluted by 500 fold with phosphate buffer (10 mM; pH 5.0)) for $24 \mathrm{~h}$. The original serum and albumin depleted were analysed with a typical shotgun proteome analysis protocol (see Supporting Information). The false positive rate was set to less than $1 \%$ for the human plasma proteome analysis, while all results were obtained in triplicate.

\section{Results and Discussion}

Preparation of the protein imprinted materials by hierarchical imprinting. During the preparation of the protein imprinted materials, MA was chosen as the functional monomer, because it could interact with the template protein through the hydrogen interactions $s^{7,14}$. Its structure analog, acrylamide, has also been used in the previous hierarchical imprinting. Herein, MAA, as the charged monomer, was also employed as the additional co-functional monomer, due to small amount charged monomer could form the electric interaction with the template to favor the formation of the recognition sites. Moreover, it has been witnessed in our previous study that better protein recognition ability could be obtained with MA or its analogs as the principal functional monomer, accompanied by a small amount of a basic or acidic co-monomer. Additionally, PDA, instead of the common methylenebisacrylamide, was used as the cross-linker to ensure the good hydrophilicity and mechanic stability for the prepared imprinted polymers ${ }^{13}$. Herein, PSA was employed as the substitute template to HSA, because its highly similarity to HSA on the sequence and structure. Furthermore, the acquirement of the PSA is much easier than that of the HSA, which would favour the large scale preparation of the molecularly imprinted materials.
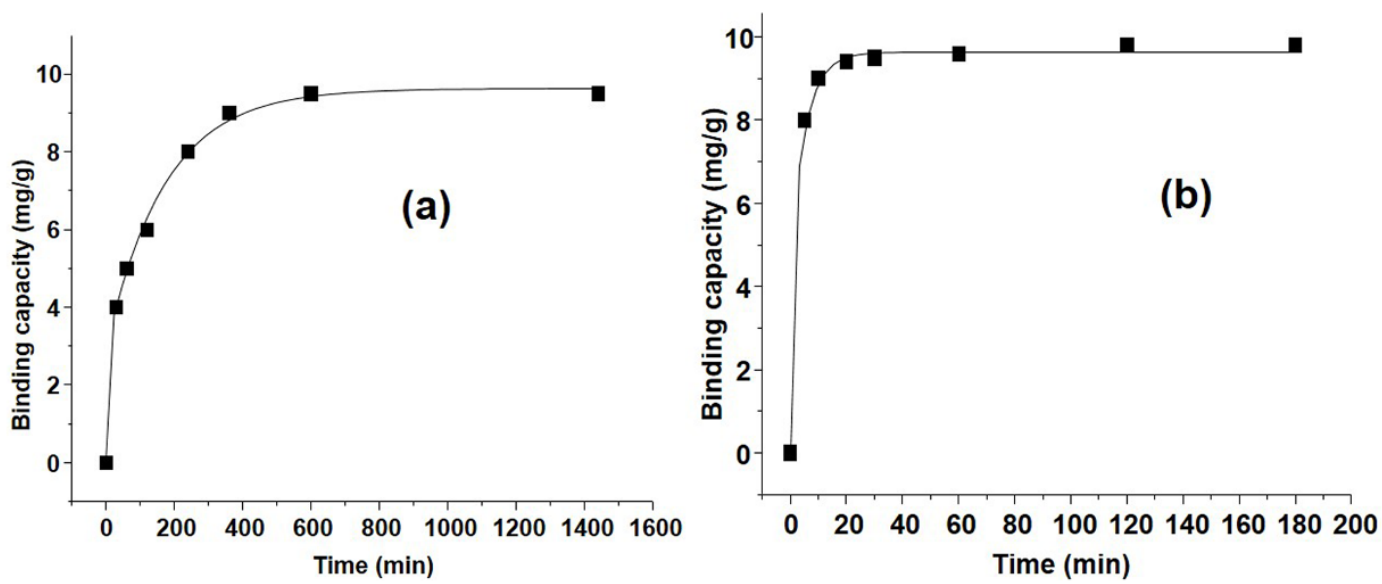

Figure 3 Binding kinetics of b-MIPs (a) and h-MIPs (b). 

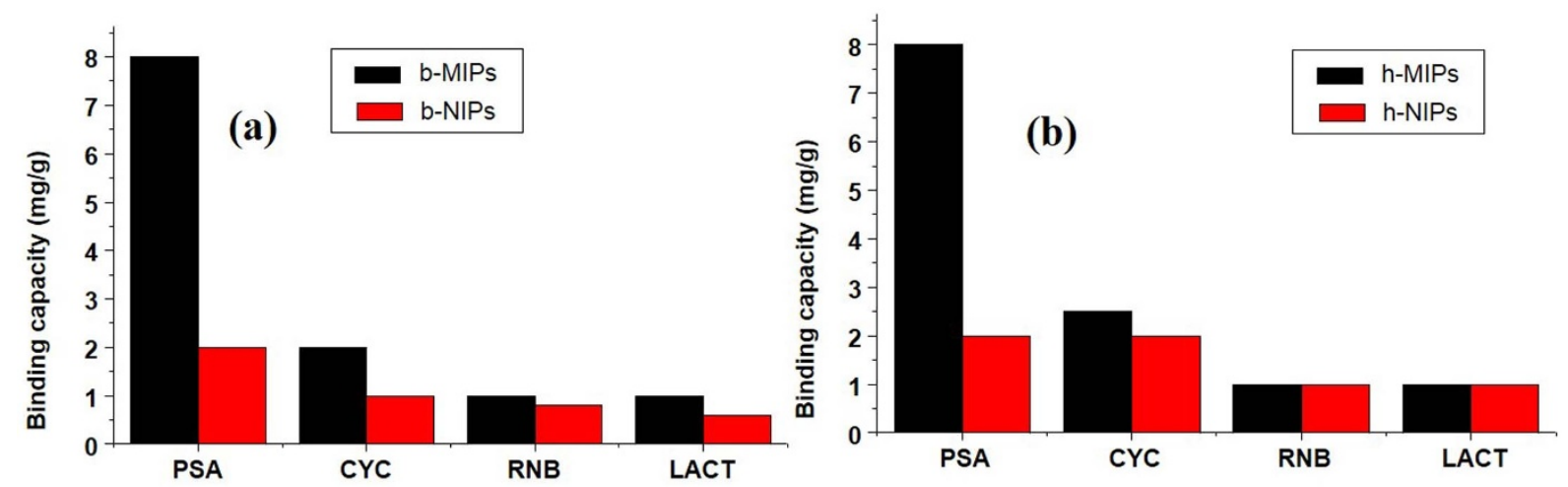

Figure $4 \mid$ Competitive binding experiment of b-MIPs and b-NIPs (a), as well as h-MIPs and h-NIPs (b).

Evaluation of the protein imprinted materials by hierarchical imprinting. The properties of MIPs and NIPs synthesized by both bulk polymerization and hierarchical imprinting were compared. For the morphology, the images of the b-MIPs, silica mold and hMIPs were shown in Fig. S1, Supporting Information. Unregulated particles, with the particle size ranged from $5.0 \mu \mathrm{m}$ to $8.0 \mu \mathrm{m}$ were observed for the b-MIPs, aggregated by smaller beads with particle size less than $2.5 \mu \mathrm{m}$. The h-MIPs were observed with spherical profile and rugged surface, with particle size about $4.8 \mu \mathrm{m}$, similar to that of the silica mold particles.

The binding capacity of b-MIPs and b-NIPs, as shown in Fig. 2, was respectively $9.4 \mathrm{mg} \mathrm{g}^{-1}$ and $2.0 \mathrm{mg} \mathrm{g}^{-1}$, and that for h-MIPs and h-NIPs was respectively $12 \mathrm{mg} \mathrm{g}^{-1}$ and $3.5 \mathrm{mg} \mathrm{g}^{-1}$. The obviously improved binding capacity of MIPs compared to NIPs should be contributed to PSA recognition sites formed by molecular imprinting.

Usually, the binding capacity of surface imprinting is lower than that of bulk materials, because imprinting sites were formed all over the bulk-imprinted MIPs, but only located on the surface of surfaceimprinted $\mathrm{MIPs}^{19}$. In this experiment, with porous silica as mold, the resultant h-MIPs were also porous, with the specific surface areas as $71.9 \mathrm{~m}^{2} / \mathrm{g}$, similar to that for b-MIPs $\left(61.8 \mathrm{~m}^{2} / \mathrm{g}\right)$. Therefore, enough recognition sites for PSA were formed on h-MIPs beads, resulting in equivalent, or even a little higher binding capacity than that of bMIPs. The swelling ratio of h-MIPs and b-MIPs were from the swelling experiments in water separately at $40^{\circ} \mathrm{C}$. The swelling ratio was calculated according to the previous publications ${ }^{20}$. They were 2.63 and 2.50 for the b-MIPs and h-MIPs.

Adsorption equilibrium rate is also an important parameter to evaluate MIPs, which reflects the time required to reach the maximum adsorption of template molecules. From the results shown in Fig. 3, it could be seen that, for b-MIPs, the adsorption kinetics increased fast in the first $60 \mathrm{~min}$, reaching about $52 \%$ of the max- imum binding capacity, then slowed down, and reached adsorption equilibrium in $600 \mathrm{~min}$. In contrast, the adsorption rate of h-MIPs increased significantly in the first $5 \mathrm{~min}$, and reached equilibrium in $20 \mathrm{~min}$, which should be contributed to the easy accessibility of the recognition sites for template in h-MIPs. Such fast mass transfer of template protein shows the promising of h-MIPs in practical applications, such as SPE and chromatography separation.

To evaluate the selectivity of MIPs, both b-MIPs and h-MIPs were subjected to a mixture of four proteins composed of PSA (pI 5.0), $\beta$ lactoglobulin (LACT, pI 5.1), basic cytochrome C (CYC, pI 10.6) and ribonuclease B (RNB, pI 8.8), including both acidic and basic competitive proteins. In addition, the PI of PSA was quite close to LACT, by which the discrimination capacity of prepared MIPs could be evaluated. As shown in Fig. 4, both b-MIPs and h-MIPs exhibit excellent selectivity for PSA, with imprinting factors as about 3.6, much higher than those for non-template proteins, with imprinting factors as 1.0 for RNB and LACT by b-MIPs and h-MIPs, 1.5 for CYC by b-NIPs, and 2.0 for CYC by h-NIPs. These results indicate that MIPs prepared by both hierarchical imprinting and bulk polymerizations are of excellent selectivity for the template protein.

For MIPs, especially with protein as the template, the synthesis reproducibility is an important issue to consider ${ }^{21}$. In our experiments, 5 batches of b-MIPs and h-MIPs were respectively synthesized, and the competitive binding experiments were performed. From the data shown in Fig. 5, it could be seen that the binding capacity reproducibility of h-MIPs for PSA is much better than that of b-MIPs. For the preparation of b-MIPs, even great care was taken in each synthesis step, it was still difficult to ensure the batch to batch reproducibility, since any small disturbance during polymerization might lead to the denature or conformation change of template protein, resulting in decreased number of specific recognition sites. In contrast, by hierarchical imprinting, the template immobilization and polymerization performed in different steps under the respect-
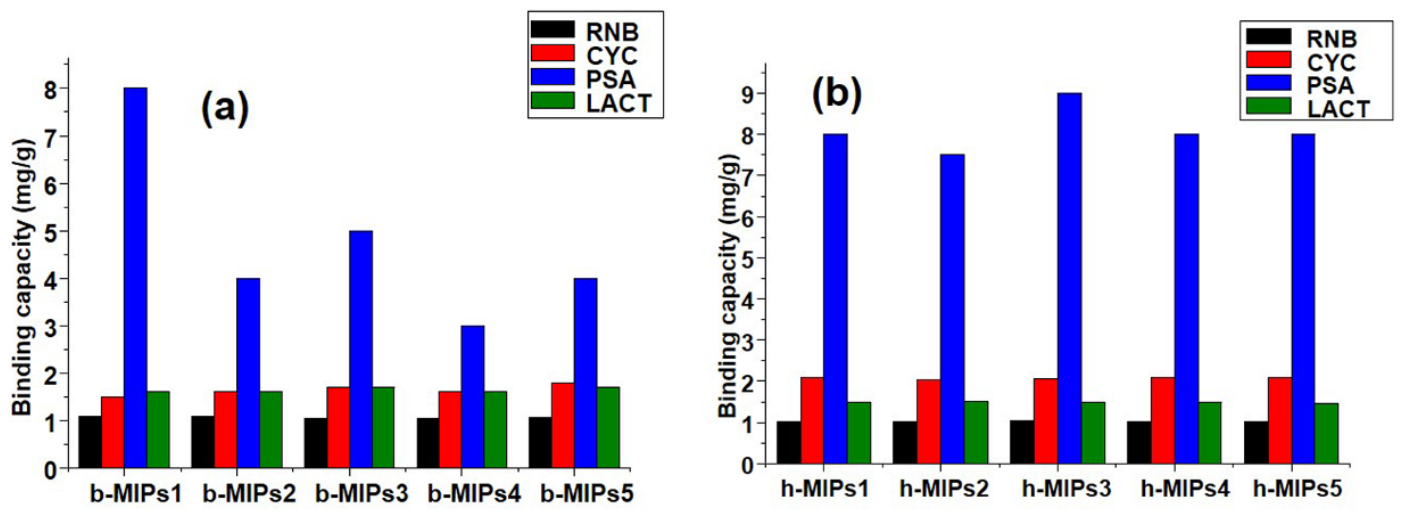

Figure 5 | Batch-to-batch reproducibility of b-MIPs (a) and h-MIPs (b). 
Table 1 Number of identified peptides from HSA and total protein groups from serum before and after HSA depletion by h-MIPs

Original serum Serum treated by MIPs Serum treated by NIPs Serum treated by antibody

Percentage of HSA peptides in total peptides (\%)

Total protein groups

$\begin{array}{llcc}37.8 & 13.9 & 42.7 & 1.9 \\ 348 & 422 & 363 & 376\end{array}$

ively optimized conditions is beneficial for keeping stable reaction solution. Further, the immobilization of PSA on silica by physical adsorption could greatly improve the conformation stability of template protein, ensuring the excellent batch-to-batch preparation reproducibility of MIPs.

Therefore, with the comprehensive consideration of selectivity, binding capacity, adsorption kinetics and preparation reproducibility, MIPs prepared by hierarchical imprinting is very applicable in practical applications.

Application of the protein imprinted materials by hierarchical imprinting. Original serum, and serum sample treated by h-MIPs, h-NIPs and Albumin Removal kit (obtained from Merck) were digested by trypsin. Then, the peptides were analyzed by $2 \mathrm{D}$ nanoSCX-RPLC-MS/MS.

From the data shown in Table 1, with the treatment of h-MIPs and Albumin Removal kit, the percentage of HSA peptides in total peptides decreased significantly. This result indicated that h-MIPs and Albumin Removal kit removed HSA in serum samples efficiently. It could be seen that with stringent filtering criteria for human plasma proteome analysis (false discovery rate less than $1 \%$ ), the identified protein group number in serum treated by h-MIPs was increased to 422 , higher than that obtained from original serum. The identified protein group number for Albumin Removal kit was 376, a little higher than that in original serum. Noticeably, protein numbers identified from h-MIPs-treated sample were much higher than that from Albumin removal kit-treated sample. The reasons should be that due to non-specific adsorption of MIPs, other high abundance proteins were also removed simultaneously with HSA. Hence, more low abundance proteins were identified in MIPs-treated sample. Because albumin cannot be removed efficiently, the identified protein group numbers of h-NIPs-treated sample were similar to original serum. The poor removal of HSA by h-NIPs demonstrated that the selective depletion of HSA by h-MIPs should be contributed to molecular imprinting.

\section{Conclusion}

In conclusion, protein imprinted materials by hierarchical imprinting was successfully prepared and, to the best of our knowledge, for the first time, as the artificial antibody, were applied to remove the highest abundance proteins from human serum. Compared with MIPs prepared by bulk polymerization, those prepared by hierarchical imprinting technique show advantages of excellent selectivity, high binding capacity, fast adsorption kinetics and good synthesis reproducibility. With PSA, the analog of HSA, the imprinted microspheres were further successfully applied for the depletion of HSA from human serum, the highest abundance protein in plasma, to improve the identification capacity of low abundance proteins. These results demonstrated that protein imprinted polymers prepared by hierarchical imprinting technique, might become really artificial antibodies for the selective depletion of high abundance protein, and further the selective enrichment of low abundance ones, especially for those without antibodies, in proteome study.

1. Whitcombe, M. J. Molecularly imprinted polymers: smart hydrogel crystal gardens. Nat. Chem. 3, 657-658 (2011).

2. Sellergren, B. Molecularly imprinted polymers: shaping enzyme inhibitors. Nat. Chem. 2, 7-8 (2010).
3. Wulff, G. \& Liu, J. Design of biomimetic catalysts by molecular imprinting in synthetic polymers: the role of transition state stabilization. Acc. Chem. Res. 45, 239-247 (2012).

4. Zeng, Z. et al. Synthetic polymer nanoparticle-polysaccharide interactions: a systematic study. J. Am. Chem. Soc. 134, 2681-2690 (2012).

5. Yoshimatsu, K. et al. Temperature-responsive "catch and release" of proteins by using multifunctional polymer-based nanoparticles. Angew. Chem. Int. Ed. 51, 2405-2408 (2012).

6. Chen, L. X., Xu, S. F. \& Li, J. H. Recent advances in molecular imprinting technology: current status, challenges and highlighted applications. Chem. Soc. Rev. 40, 2922-2942 (2011).

7. Whitcombe, M. J. et al. A. The rational development of molecularly imprinted polymer-based sensors for protein detection. Chem. Soc. Rev. 40, 1547-1571 (2011).

8. Zheng, C. et al. A Selective Artificial Enzyme Inhibitor Based on NanoparticleEnzyme Interactions and Molecular Imprinting. Adv. Mater. 25, 5922-5927 (2013).

9. Ma, Y., Pan, G. Q., Zhang, Y., Guo, X. Z. \& Zhang, H. Q. Narrowly Dispersed Hydrophilic Molecularly Imprinted Polymer Nanoparticles for Efficient Molecular Recognition in Real Aqueous Samples Including River Water, Milk, and Bovine Serum. Angew. Chem. Int. Ed. 52, 1511-1514 (2013).

10. Shinde, S., Bunschoten, A., Kruijtzer, J. A. W., Liskamp, R. M. J. \& Sellergren, B. Imprinted Polymers Displaying High Affinity for Sulfated Protein Fragments. Angew. Chem. Int. Ed. 51, 8326-8329 (2012).

11. Liu, J. et al. Preparation of a new type of affinity materials combining metal coordination with molecular imprinting. Chem. Commun. 47, 3969-3971 (2011).

12. Titirici, M. M., Hall, A. J. \& Sellergren, B. Hierarchical Imprinting Using Crude Solid Phase Peptide Synthesis Products as Templates. Chem. Mater. 15, 822-824 (2003).

13. Li, Q. R. et al. Transferrin recognition based on a protein imprinted material prepared by hierarchical imprinting technique. Microchim. Acta 180, 1379-1386 (2013).

14. Yang, K. G., Zhang, L. H., Liang, Z. \& Zhang, Y. K. Protein-imprinted materials: rational design, application and challenges. Anal. Bioanal. Chem. 403, 2173-2183 (2012).

15. Kelloff, G. J. \& Sigman, C. C. Cancer biomarkers: selecting the right drug for the right patient. Nat. Rev. Drug Discov. 11, 201-214 (2012)

16. Yang, B. et al. Identification of cross-linked peptides from complex samples. Nat. Methods 9, 904-906 (2012).

17. Blow, N. Proteins and proteomics: life on the surface. Nat. Methods 6, 389-392 (2009).

18. Issaq, H. J., Xiao, Z. \& Veenstra, T. D. Serum and plasma proteomics. Chem. Rev. 107, 3601-3620 (2007).

19. Pan, G. Q., Zhang, Y., Ma, Y., Li, C. X. \& Zhang, H. Q. Efficient One-Pot Synthesis of Water-Compatible Molecularly Imprinted Polymer Microspheres by Facile RAFT Precipitation Polymerization. Angew. Chem. Int. Ed. 50, 11731-11734 (2011).

20. Li, S. Y., Lamminmaki, J. \& Hanhi, K. Improvement of mechanical properties of rubber compounds using waste rubber/virgin rubber. Polym. Eng. Sci. 45, 1239-1246 (2005).

21. Shen, X., Zhou, T. \& Ye, L. Molecular imprinting of protein in Pickering emulsion. Chem. Commun. 48, 8198-8200 (2012).

\section{Acknowledgments}

This work was supported by the National Basic Research Program of China (2012CB910601 and 2013CB911201), the National Natural Science Foundation (21190043 and 21375128), the Creative Research Group Project by NSFC (21321064), the National High Technology Research and Development Program of China (2012AA020202), and the analytical method innovation program of MOST (2012IM030900).

\section{Author contributions}

J.L., L.Z., Z.L., Z.L. and Y.Z. designed research. J.L., Q.D., D.T. and K.Y. performed research. J.L. and K.Y. analyzed data. J.L., Q.D. and K.Y. wrote the manuscript.

\section{Additional information}

Supplementary information accompanies this paper at http://www.nature.com/ scientificreports

Competing financial interests: The authors declare no competing financial interests. 
How to cite this article: Liu, J.X. et al. Preparation of protein imprinted materials by hierarchical imprinting techniques and application in selective depletion of albumin from human serum. Sci. Rep. 4, 5487; DOI:10.1038/srep05487 (2014).

(c) (i) (2) (2) This work is licensed under a Creative Commons Attribution-NonCommercialShareAlike 4.0 International License. The images or other third party material in this article are included in the article's Creative Commons license, unless indicated otherwise in the credit line; if the material is not included under the Creative Commons license, users will need to obtain permission from the license holder in order to reproduce the material. To view a copy of this license, visit http:// creativecommons.org/licenses/by-nc-sa/4.0/ 